\title{
Perímetro do abdômen é o melhor indicador antropométrico de riscos para doenças cardiovasculares
}

\section{Abdominal perimeter is the best anthropometric risk indicator of cardiovascular diseases}

\author{
Maria Fátima Glaner \\ Andreia Pelegrini 2, \\ Thales Boaventura Rachid Nascimento
}

\begin{abstract}
1 Universidade Estadual de Londrina. Centro de Educação Física e Esporte. Departamento de Educação Física. Londrina, Paraná, Brasil.

2 Universidade Federal de Santa Catarina. Centro de Desportos. Programa de Pós-Graduação em Educação Física. Florianópolis, SC. Brasil.
\end{abstract}

3 Universidade Estadual do Oeste do Paraná. Centro de Ciências Humanas, Educação e Letras. Colegiado de Educação Física. Marechal Cândido Rondon, PR.Brasil.

4 Bolsista da Coordenação de Aperfeiçoamento de Pessoal de Nível Superior-CAPES. Brasília, DF. Brasil.

Recebido em 27/04/10 Revisado em 15/06/10 Aprovado em 22/07/10
Resumo - Os objetivos deste estudo foram: a) verificar a prevalência de gordura corporal inadequada por meio do índice de massa corpórea (IMC), perímetro do abdômen (PAB) e gordura corporal relativa $(\mathrm{G} \%)$, e indicadores bioquímicos de risco para doenças cardiovasculares; b) analisar quais indicadores de gordura corporal refletem melhor os fatores bioquímicos. Em 125 homens foram mensurados: IMC, G\%, PAB, glicemia, triglicerídeos, colesterol total, lipoproteínas de alta densidade (HDL) e lipoproteínas de baixa densidade (LDL). Mais da metade da amostra (56,8\%) apresentou baixos valores de HDL e elevados valores de IMC (58,4\%) e G\% (53,6\%). Menor prevalência de sujeitos foi observada com elevados valores de LDL (40,8\%), PAB (38,4\%), triglicerídeos (34,8\%), glicemia (27,2\%) e colesterol total (15,2\%). O PAB associou-se com quatro indicadores bioquímicos, enquanto que a $\mathrm{G} \%$ com três e o IMC somente com dois. A prevalência dos fatores de risco foi elevada. $\mathrm{O}$ PAB $>92 \mathrm{~cm}$ mostrou-se o melhor indicador de gordura, apontando que os homens estão expostos a elevados valores de LDL, colesterol total, triglicerídeos e glicemia.

Palavras-chave: Cardiopatias; Obesidade; Dislipidemias; Antropometria; Topografia da Gordura.

Abstract - The objectives of this study were: a) to determine the prevalence of inadequate body fat determined based on body mass index (BMI), abdominal perimeter (AP) and relative body fat (\%F), and biochemical indicators of cardiovascular risk, and b) to examine which body fat indicators better reflect the biochemical factors. BMI, \%F, AP, glucose, triglycerides, total cholesterol, high-density lipoprotein (HDL), and low-density lipoprotein (LDL) were measured in 125 men. More than half the sample (56.8\%) had low HDL and high BMI (58.4\%) and \%F (53.6\%). There was a lower percentage of subjects with elevated LDL (40.8\%), AP (38.4\%), triglycerides (34.8\%), glucose (27.2\%), and total cholesterol (15.2\%). AP was associated with four biochemical markers, whereas $\% \mathrm{~F}$ was associated with three and BMI with only two. The prevalence of risk factors was high. An AP $>92 \mathrm{~cm}$ proved to be the best fat indicator, demonstrating that men are exposed to high levels of LDL, total cholesterol, triglycerides, and glucose. Key words: Heart Diseases; Obesity; Dyslipidemias; Anthropometry; Fat Topography. 


\section{INTRODUÇÃO}

O excesso de gordura corporal é fator de risco para o desenvolvimento de doenças crônicas não-transmissíveis, como as cardiovasculares, o diabetes e a hipertensão arterial ${ }^{1 .} \mathrm{O}$ acúmulo excessivo de gordura na região central do corpo é um dos indicadores para o diagnóstico da síndrome metabólica ${ }^{2}$ e está relacionado ao surgimento de dislipidemias ${ }^{3}$ e ao diabetes ${ }^{4}$.

Elevados valores de triglicerídeos, colesterol total, lipoproteínas de baixa densidade (LDL) e glicemia em jejum, e baixos valores de lipoproteínas de alta densidade (HDL), são fatores bioquímicos de risco para o desenvolvimento de doenças cardíacas $^{5,6}$. Estudos ${ }^{4,-9}$ demonstram o relacionamento de diferentes indicadores de gordura corporal, como o índice de massa corporal (IMC), o perímetro do abdômen (PAB) e a gordura corporal relativa (G\%), com os fatores bioquímicos de risco para as doenças cardiovasculares, no entanto, os resultados são contraditórios. Os pontos de corte destes indicadores, também, podem não ser os mais apropriados para determinados grupos populacionais ${ }^{10,11}$. No caso da $\mathrm{G} \%$, a técnica usada para sua quantificação deve ser a mesma que derivou os pontos de corte. Por um lado, as técnicas de campo, como a antropometria, são baratas e realizáveis em larga escala. Por outro lado, as técnicas laboratoriais são relativamente mais acuradas, porém com menor aplicabilidade, como a absortometria de raio $\mathrm{X}$ de dupla energia (DXA).

Os estudos previamente citados, envolvendo fatores bioquímicos de risco cardiovascular e indicadores de gordura, são, em grande parte, realizados em populações homogêneas e de raça pouco mesclada. Existe a necessidade de evidências envolvendo amostras da população brasileira, a qual é predominantemente multirracial. Diante do exposto, e em razão dos fatores bioquímicos de risco serem processos assintomáticos, que podem permanecer assim por longos períodos, os objetivos deste são: a) verificar a prevalência de gordura corporal inadequada por meio do IMC, PAB e G\%, e indicadores bioquímicos de risco para doenças cardiovasculares; b) analisar quais indicadores de gordura corporal refletem melhor os fatores bioquímicos.

\section{PROCEDIMENTOS METODOLÓGICOS}

\section{Amostra}

A amostra inicial foi composta por 200 funcionários de uma metalúrgica, todos voluntários, que atuam na administração, manufatura, transporte e venda do metal. Foram excluídos da amostra os sujeitos que se enquadraram em uma ou mais das categorias seguintes: não seguiram o jejum alimentar de $12 \mathrm{~h}$; usavam repositores hormonais orais ou injetáveis, remédios antilipêmicos e para o controle do diabetes; fumavam; ingeriam bebidas alcóolicas de forma regular (mais do que dois dias na semana); apresentaram mal estar agudo como vertigens ou febre, ou crônico, como esclerose das veias dos braços; possuiam triglicérides $>400 \mathrm{mg} /$ dL. A amostra final foi composta por 125 homens, com idade média de 35,6 (desvio padrão= 10,7) anos. A maioria deles é advinda da região Nordeste (48,3\%) seguida da Centro-Oeste (35,3\%); 15,3\% são da região Sudeste e 1,1\% da região Sul do Brasil.

\section{Procedimentos para coleta}

Durante uma aula de ginástica laboral, todos os funcionários da empresa foram informados previamente sobre o propósito em questão e orientados para seguir o jejum alimentar de $12 \mathrm{~h}$. Todos que concordaram em participar assinaram um termo de consentimento livre e esclarecido que detalhava os procedimentos que seriam realizados para a coleta de dados, e que lhes garante o total anonimato. Esta pesquisa foi aprovada pelo Comitê de Ética e Pesquisa da Universidade Católica de Brasília (Parecer no 04/2005), consoante à Declaração de Helsinki.

\section{Coleta de dados}

A massa corporal (MC) e a estatura (ES) foram mensuradas em uma balança Filizola (escala de 0,1 kg) com estadiômetro acoplado (escala de 0,5 $\mathrm{cm})$. $O$ índice de massa corporal foi obtido pela expressão: $\mathrm{IMC}=\mathrm{MC}_{\mathrm{kg}} / \mathrm{ES}_{\mathrm{m}}{ }^{2} \cdot \mathrm{O}$ perímetro do abdômen (PAB) foi medido, com uma fita métrica, a $2,5 \mathrm{~cm}$ acima da cicatriz umbilical. Todas as medidas antropométricas seguiram os procedimentos descritos em Petroski ${ }^{12}$.

A gordura corporal total, expressa em termos relativos $(G \%)$, foi obtida por absortometria de raio $\mathrm{X}$ de dupla energia (DXA). Todos os procedimentos descritos no manual do equipamento (Lunar, DPX-IQ, software v. 4.7e) foram seguidos rigorosamente. As medidas antropométricas e análise dos exames de DXA para obter a $G \%$ foram feitas por um único avaliador, cujo erro técnico de medida é inferior a $1 \%$ para as variáveis antropométricas e, $<1,6 \%$ (coeficiente de variação) para a G\% via DXA.

Para obter os valores de glicemia, triglicerídeos, colesterol total e HDL foi coletado sangue em uma das veias da fossa antecubital do braço. 
Aproximadamente, $3 \mathrm{~mL}$ de sangue foram coletados em cada tubo a vácuo (Bencton Dickinson), um com EDTA e outro com fluoreto. Os tubos com o sangue foram colocados em uma caixa térmica com gelo. $\mathrm{O}$ tempo entre a coleta e a centrifugação não excedeu a $2 \mathrm{~h}$. Para as análises bioquímicas, foi usado um espectrofotômetro semi-automático da Bioplus, modelo BIO-2000. Os reagentes bioquímicos usados foram da marca Doles. Foram seguidos todos os procedimentos descritos pelos fabricantes do espectrofotômetro e dos reagentes. Os valores das LDL foram obtidos usando os resultados de triglicerídeos (TG), colesterol total (CT) e HDL, a partir da fórmula $[\mathrm{LDL}=\mathrm{CT}-(\mathrm{HDL}+\mathrm{TG} \div 5)] \mathrm{de}$ Friedewald, Levy e Fredrickson ${ }^{13}$.

A categorização considerada normal, usada nas análises estatísticas, foi: $\mathrm{HDL} \geq 40 \mathrm{mg} / \mathrm{dL}$; LDL $\geq 100 \mathrm{mg} / \mathrm{dL}$; colesterol total $\leq 200 \mathrm{mg} / \mathrm{dL}$; triglicerídeos $\leq 150 \mathrm{mg} / \mathrm{dL}$; glicemia $\leq 100 \mathrm{mg} / \mathrm{dL}$ e $P A B \leq 92 \mathrm{~cm}$, conforme a International Diabetes Federation - IDF ${ }^{14}$. Esta sugere: para sul americanos deve ser adotado o mesmo $(90 \mathrm{~cm})$ ponto de corte que para os sul asiáticos; para europeus $94 \mathrm{~cm}$. Devido as características da amostra, optou-se por um ponto de corte intermediário, $92 \mathrm{~cm}$. Ainda, o IMC foi considerado normal, sendo $\leq 25 \mathrm{~kg} / \mathrm{m}^{2}$, conforme recomendação da Organização Mundial de Saúde ${ }^{15}$, e a $G \% \leq 19 \%{ }^{16}$. Valores opostos a estes compuseram a categoria elevada.

\section{Análise estatística}

Todas as variáveis, exceto massa corporal e estatura, foram trabalhadas de forma dicotômica: normal e elevado ou baixo. Utilizou-se o teste qui-quadrado para comparar as freqüências entre as categorias das variáveis antropométricas e os indicadores de risco cardiovascular. A associação entre o desfecho/acontecimento (indicadores de risco cardiovascular) e as variáveis independentes/fator (antropometria) foi analisada por meio da análise de regressão logística. Em todas as análises foi fixado o nível de significância de 5\% ( $\leq \leq 0,05$ ou IC 95\%). Para as análises, foi usado o Statistical Package for the Social Sciences - versão 15.0, com licença de uso.

\section{RESULTADOS}

A tabela 1 apresenta as características antropométricas, G\% e bioquímicas da amostra. Os valores médios do IMC e da G\% indicam um quadro de sobrepeso, enquanto o HDL está abaixo do recomendado. Os valores médios do PAB e demais variáveis bioquímicas estão conforme os valores de referência normais ${ }^{14}$.
Tabela 1. Características antropométricas e bioquímicas da amostra $(n=125)$.

\begin{tabular}{lc}
\hline Variáveis & Médias \\
\hline Massa corporal $(\mathrm{kg})$ & $72,5 \pm 10,9$ \\
Estatura $(\mathrm{cm})$ & $168,2 \pm 7,0$ \\
IMC $\left(\mathrm{kg} / \mathrm{m}^{2}\right)$ & $25,8 \pm 3,5$ \\
PAB $(\mathrm{cm})$ & $86,8 \pm 10,2$ \\
G\% & $20,5 \pm 8,4$ \\
HDL $(\mathrm{mg} / \mathrm{dL})$ & $39,1 \pm 10,8$ \\
LDL $(\mathrm{mg} / \mathrm{dL})$ & $97,9 \pm 35,0$ \\
Colesterol total $(\mathrm{mg} / \mathrm{dL})$ & $165,5 \pm 38,5$ \\
Triglicerídeos $(\mathrm{mg} / \mathrm{dL})$ & $145,1 \pm 87,7$ \\
Glicemia $(\mathrm{mg} / \mathrm{dL})$ & $94,7 \pm 15,9$ \\
\hline
\end{tabular}

IMC: índice de massa corporal; PAB: perímetro do abdômen; G\%: gordura corporal relativa; HDL: lipoproteína de alta densidade; LDL: lipoproteína de baixa densidade.

As associações entre os indicadores de gordura e os fatores de risco para as doenças cardiovasculares estão na tabela 2. Foi verificada associação entre: 0 IMC com a LDL $\left(\chi^{2}=5,27 ; \mathrm{p}=0,017\right)$ e os triglicerí$\operatorname{deos}\left(\chi^{2}=8,84 ; \mathrm{p}=0,002\right)$; o perímetro do abdômen com a LDL $\left(\chi^{2}=5,76 ; \mathrm{p}=0,014\right)$, colesterol total $\left(\chi^{2}=\right.$ $8,54 ; p=0,004)$, triglicerídeos $\left(\chi^{2}=10,5 ; p=0,001\right)$ e glicemia $\left(\chi^{2}=4,18 ; p=0,034\right)$; a $G \%$ com a LDL $\left(\chi^{2}=10,0 ; p=0,001\right)$, colesterol total $\left(\chi^{2}=11,6 ; p\right.$ $<0,0001)$ e triglicerídeos $\left(\chi^{2}=11,7 ; p=0,001\right)$. Mais da metade da amostra apresentou valores elevados de IMC (58,4\%), G\% (53,6\%) e HDL $(56,8 \%)$; seguido da LDL (40,8\%), PAB (38,4\%), triglicerídeos $(34,8 \%)$, glicemia $(27,2 \%)$ e colesterol total $(15,2 \%)$. Maior proporção de indivíduos com IMC $>25 \mathrm{~kg} / \mathrm{m}^{2}$ tinha LDL e triglicerídeos elevados. Os indivíduos com $\mathrm{PAB}>92 \mathrm{~cm}$, também, em maior proporção, tiveram LDL, colesterol total, triglicerídeos e glicemia elevados. Entre aqueles com $\% \mathrm{G}>19 \%$, encontrou-se maior proporção de sujeitos com LDL, colesterol total e triglicerídeos elevados.

$\mathrm{Na}$ tabela 3, estão apresentadas as associações (regressão logística), do IMC, PAB e G\% com os demais fatores de risco para as doenças cardiovasculares. Encontrou-se associação entre o IMC com a LDL [OR=2,40; IC (95\%): 1,13-5,11; $\leq 00,05]$ e os triglicerídeos [OR=3,24; IC (95\%): 1,47-7,16; $\mathrm{p} \leq 0,05]$. Houve associação entre o perímetro do abdômen e, o LDL [OR=2,46; IC (95\%): 1,17-5,16; $\leq \leq 0,05]$, o colesterol total [OR= 4,4; IC (95\%): 1,54-12,54; $\mathrm{p} \leq 0,05]$, os triglicerídeos [OR=3,43; IC (95\%): 1,60-7,33; p $\leq 0,05]$ e a glicemia [OR=2,29; $\operatorname{IC}(95 \%): 1,03-5,11$; $\mathrm{p} \leq 0,05]$. A G\% associou-se com as variáveis LDL [OR=3,33; IC (95\%): 1,56-7,11; $\mathrm{p} \leq 0,05]$, colesterol total [OR=9,52 (95\%): 2,10-43,27; $\mathrm{p} \leq 0,05]$ e triglicerídeos [OR=3,79; IC (95\%): 1,73-8,27; p s0,05]. 
Tabela 2. Associação do índice de massa corporal (IMC), perímetro do abdômen (PAB), gordura corporal relativa (G\%) e os indicadores bioquímicos de risco para as doenças cardiovasculares.

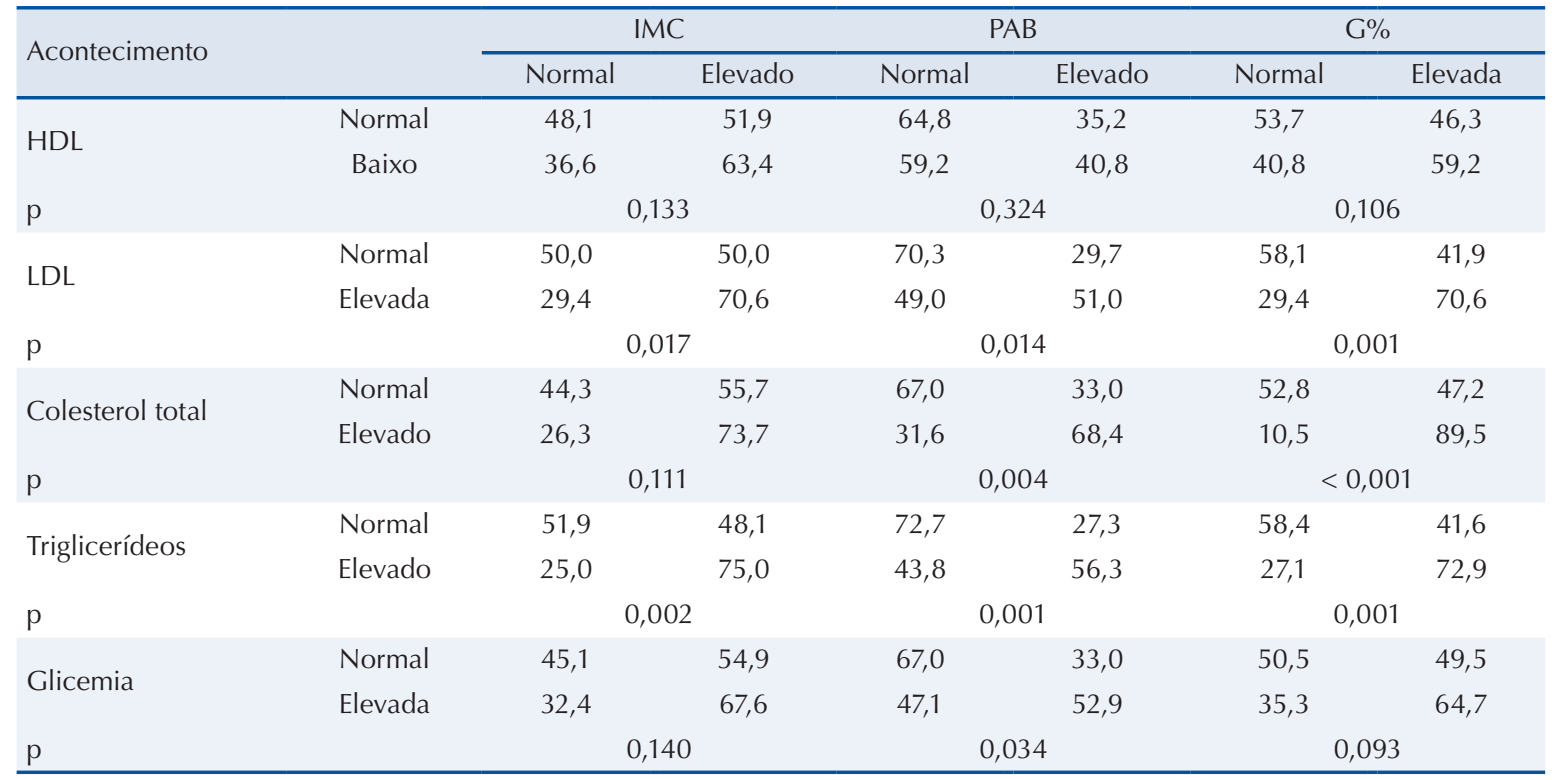

HDL: lipoproteína de alta densidade; p; nível de significância; LDL: lipoproteína de baixa densidade.

Tabela 3. Associação entre o índice de massa corporal (IMC), perímetro do abdômen (PAB) e gordura corporal relativa (G\%) com os indicadores bioquímicos de risco para as doenças cardiovasculares.

\begin{tabular}{lcccc}
\hline \multirow{2}{*}{ Acontecimento } & Fator & \multicolumn{3}{c}{ Odds ratio (intervalo de confiança 95\%) } \\
\cline { 3 - 5 } HDL & Normal & IMC & PAB & 1 \\
& Baixo & 1 & 1 & G\% \\
LDL & Normal & $1,61(0,78-3,30)$ & $1,27(0,61-2,65)$ & $1,68(0,82-3,43)$ \\
& Elevada & 1 & 1 & 1 \\
Colesterol total & Normal & $2,40(1,13-5,11)^{*}$ & $2,46(1,17-5,16)^{*}$ & $3,33(1,56-7,11)^{*}$ \\
& Elevado & 1 & 1 & 1 \\
Triglicerídeos & Normal & $2,23(0,75-6,64)$ & $4,40(1,54-12,54)^{*}$ & $9,52(2,10-43,27)^{*}$ \\
& Elevado & 1 & 1 & 1 \\
Glicemia & Normal & $3,24(1,47-7,16)^{*}$ & $3,43(1,60-7,33)^{*}$ & $3,79(1,73-8,27)^{*}$ \\
& Elevada & $1,72(0,75-3,93)$ & 1 & 1 \\
\end{tabular}

HDL: lipoproteína de alta densidade; LDL: lipoproteína de baixa densidade. * p $\leq 0,05$.

\section{DISCUSSÃO}

Os objetivos deste estudo foram: a) verificar a prevalência de gordura corporal inadequada por meio do IMC, PAB e G\%, e indicadores bioquímicos de risco para doenças cardiovasculares; b) analisar quais indicadores de gordura corporal refletem melhor os fatores bioquímicos. Constatou-se que mais da metade da amostra $(56,8 \%)$ apresentou baixos valores de HDL e elevados valores de IMC $(58,4 \%)$ e G\% (53,6\%). Menor prevalência de sujeitos foi observada com elevados valores de LDL $(40,8 \%)$, PAB $(38,4 \%)$, triglicerídeos $(34,8 \%)$, glicemia $(27,2 \%)$ e colesterol total $(15,2 \%)$. As prevalências dos fatores de risco correspondentes aos analisados em outros estudos são inferiores ${ }^{17,18}$ e superiores ${ }^{19,20}$, inclusive em relação a siderúrgicos e metalúrgicos ${ }^{21}$. Isto retrata a grande variabilidade entre amostras de diferentes regiões do Brasil, mesmo com ocupações similares. A comparação com outros estudos/amostras é afetada por fatores individuais diversificados, além dos diferentes pontos de corte usados em cada estudo. Hábitos alimentares, características étnicas e genéticas podem influenciar os fatores de risco para doenças cardiovasculares. Por essa razão, as prevalências e associações constatadas no presente estudo representam uma população masculina com características semelhantes às da amostra em questão. Ainda, reporta-se a necessidade de planejamento de ações direcionadas/específicas à prevenção e ao monitoramento destes fatores de risco, considerando as diferenças demográficas, sócioeconômicas e de 
estilo de vida ${ }^{22,23}$. Consequentemente, o panorama das doenças cardiovasculares pode ser modificado drasticamente nas próximas cinco décadas ${ }^{22}$.

Inicialmente, os resultados demonstram associação entre os indicadores de gordura corporal e bioquímicos, conforme evidenciado em outros estudos $^{1,4,20}$. De modo geral, indivíduos com IMC $>25 \mathrm{~kg} / \mathrm{m}^{2}, \mathrm{G} \%>19 \%$ e PAB > $92 \mathrm{~cm}$ apresentam um maior risco de ser acometidos por doenças cardiovasculares. Proporções significativas de sujeitos com estas características antropométricas apresentaram elevados valores de LDL, triglicerídeos, colesterol total e glicemia. No entanto, as associações entre as frequências dos indicadores antropométricos e bioquímicos apontam que o PAB é o melhor indicador, seguido da G\%, ficando o IMC por último. Em outro estudo ${ }^{24}$, com delineamento diferenciado do presente, foi evidenciado que o PAB, medido 2,5cm acima da cicatriz umbilical, explicou 78\% da G\% abdominal, medida por DXA, em homens. Na mesma amostra, as dobras cutâneas abdominal (67\%), suprailíaca (53\%) e axilar média (66\%) explicaram a $\mathrm{G} \%$ abdominal em menor magnitude.

$\mathrm{OPAB}$ associou-se com quatro, dos cinco indicadores bioquímicos, enquanto que a $\mathrm{G} \%$ com três e o IMC somente com dois. Estes resultados corroboram as evidências existentes $7,9,20,25$. O PAB $(>90 \mathrm{~cm})$ também tem se mostrado um preditor independente de ocorrência de desfechos como angina, intervenção coronária percutânea e óbito, em homens após intervenção coronária percutânea, enquanto o IMC não foi preditor em nenhum dos $\operatorname{sexos}^{26}$. A gordura acumulada no tronco, medida por DXA, também explicou diretamente e significativamente o colesterol total, triglicerídeos, HDL e LDL, enquanto a G\% não apresentou a mesma capacidade ${ }^{27}$. Os resultados obtidos no presente estudo, mais as evidências reportadas em outros, apontam que uma medida antropométrica simples - PAB, se mostra mais eficiente do que a $G \%$ obtida por um procedimento laboratorial, de custo elevado e pouca aplicabilidade.

Em adição, os resultados da análise logística mostram que o IMC $>25 \mathrm{~kg} / \mathrm{m}^{2}$ duplica as chances para apresentar LDL elevado e triplica para os triglicerídeos. A G\% >19\% indica grande potencial para elevação do colesterol total $(9,52)$, triglicerídeos $(3,79)$ e LDL $(3,33)$; enquanto, o PAB $>92 \mathrm{~cm}$ duplica as chances para apresentar as LDL e glicemia elevadas, triplica para os triglicerídeos e quadruplica para colesterol total elevados. Estes fatores de risco se manifestam tanto em homens que estão nos limiares inferiores, dos pontos de corte, como nos que estão acima ${ }^{16}$.
O PAB foi o único indicador de gordura corporal que se associou com a glicemia, reforçando o papel determinante do acúmulo de gordura na região central do corpo e também na gênese do diabetes mellitus $2^{4,9,28}$.

No Brasil, tem-se observado o uso frequente do ponto de corte $>102 \mathrm{~cm}$ para o PAB, sendo um dos critérios para o diagnóstico da síndrome metabólica ${ }^{2}$ A $\mathrm{IDF}^{14}$ recomenda para o mesmo propósito PAB >94 $\mathrm{cm}$ para homens europeus, e $>90 \mathrm{~cm}$ para homens sul-asiáticos/chineses, sul-americanos/africanos e japoneses. Como a amostra deste estudo é miscigenada, optou-se por um ponto de corte intermediário (PAB >92 cm). Evidências em outros estudos dão suporte à utilização de um ponto de corte inferior a $102 \mathrm{~cm}^{9,25,26}$. Indivíduos com maior propensão ao surgimento de alterações metabólicas, em razão do acúmulo de gordura na região central do corpo, poderiam ser identificados em estágios mais precoces quando usado o PAB $>92 \mathrm{~cm}$. Em oposição, se usado o PAB >102 cm, sujeitos podem ser identificados em estágios mais avançados, favorecendo possíveis complicações de maior gravidade.

A principal limitação do presente estudo deve-se a grande diversidade étnica (miscigenação) da amostra, característica de Brasília - DF, por agregar em curto espaço de tempo, pessoas oriundas de diferentes regiões do Brasil, pode gerar interpretações menos acuradas. Tal fato se processa pela particularidade de cada etnia, que pode refletir: quantidades e deposição de gordura corporal diferentes para uma mesma medida, quando comparada a outras etnias ${ }^{29}$; distintos hábitos alimentares e comportamentos sócioculturais, como a prática de exercícios físicos. Agregado a isto, reforça-se a necessidade de mais estudos buscando identificar/ estabelecer pontos de corte mais acurados, para os diferentes fatores de risco cardiovascular, em função da diversidade étnico-cultural da população brasileira. Por outro lado, Brasília - DF, é um nicho brasileiro que favorece, em uma amostragem, uma representação nacional, possibilitando, desta forma, uma representação mais aproximada do que seria uma evidência obtida em uma única amostra oriunda das diferentes regiões do país.

\section{CONCLUSÃO}

Os resultados obtidos mostram que a prevalência dos fatores de risco, gordura corporal inadequada e fatores bioquímicos, foi alta. O PAB >92 cm mostrou-se o melhor indicador em relação à $\mathrm{G} \%$ 
$>19 \%$ e ao IMC $>25 \mathrm{~kg} / \mathrm{m}^{2}$, apontando que os homens estão expostos a apresentar quatro fatores elevados (LDL, colesterol total, triglicerídeos e glicemia), dos cinco analisados.

\section{REFERÊNCIAS BIBLIOGRÁFICAS}

1. Burke GL, Bertoni AG, Shea S, Tracy R, Watson KE, Blumenthal RS, et al. The impact of obesity on cardiovascular disease risk factors and subclinical vascular disease. Arch Intern Med 2008;168(9):928-35.

2. National Cholesterol Education Program. Third report of the national cholesterol education program - NCEP expert panel on detection, evaluation, and treatment of high blood cholesterol in adults (Adult Treatment Panel III) final report. Circulation 2002;106(25):3143.

3. Nguyen-Duy T-B, Nichaman MZ, Church TS, Blair $\mathrm{SN}$, Ross R. Visceral fat and liver fat are independent predictors of metabolic risk factors in men. Am J Physiol Endocrinol Metab 2003;284(6):1065-71.

4. Janssen I, Katzmarzyk PT, Ross R. Body mass index, waist circumference, and health risk. Arch Intern Med 2002;162(18):2074-2079.

5. Sarwar N, Danesh, J, Eiriksdottir G, Sigurdsson G, Wareham N, Bingham S, et al. Triglycerides and the risk of coronary heart disease. Circulation 2007;115(4):450-8.

6. Sharrett AR, Ballantyne CM, Coady SA, Heiss G, Sorlie PD, Catellier D, et al. Coronary heart disease prediction from lipoprotein cholesterol levels, triglycerides, lipoprotein(a), apolipoproteins A-I and B, and HDL density subfractions: the atherosclerosis risk in communities (ARIC) study. Circulation 2001;104(10):1108-13.

7. Canoy D, Boekholdt SM, Wareham N, Luben R, Welch A, Bingham S, et al. Body fat distribution and risk of coronary heart disease in men and women in the European prospective investigation into Cancer and Nutrition in Norfolk Cohort: a population-based prospective study. Circulation 2007;116(25):2933-43.

8. $\mathrm{Hu}, \mathrm{G}$, Tuomilehto J, Silventoinen, K, Sarti C, Männistö S, Jousilahti P. Body mass index, waist circumference, and waist-hip ratio on the risk of total and type-specific stroke. Arch Intern Med 2007;167(13)1420-7.

9. Wang Y, Rimm EB, Stampfer MJ, Willett WC, Hu FB. Comparison of abdominal adiposity and overall obesity in predicting risk of type 2 diabetes among men. Am J Clin Nutr 2005;8(3):555-63.

10. Stevens J, Cai J, Juhaeri, Thun MJ, Williamson DF, Wood JL. Consequences of the use of different measures of effect to determine the impact of age on the association between obesity and mortality. Am J Epidemiol 1999;150(4):399-407.

11. Stranges S, Trevisan M, Dorn JM, Dmochowski J, Donahue RP. Body Fat distribution, liver enzymes, and risk of hypertension: evidence from the Western New York study. Hypertension 2005;46(5):1186-93.

12. Petroski EL. editor. Antropometria: técnicas e padronizações. 2a ed. Porto Alegre: Palotti, 2003.

13. Friedewald WT, Levy RI, Fredrickson DS. Estimation of the concentration of low-density lipoprotein cholesterol in plasma without use of preparative ultracentrifuge. Clin Chem 1972;18(6):499-502.
14. International Diabetes Federation - IDF. The IDF consensus worldwide definition of the metabolic syndrome. Available from: http://www.idf.org/. [2009 jul 12].

15. World Health Organization - WHO. Diet, nutrition and prevention of chronic diseases. Available from: $<$ http://whqlibdoc.who.int/trs/who_trs_916.pdf> [2010 jun 22].

16. Glaner MF, Lima WA, Borysiuk Z. Body fat deposition and risk factors of cardiovascular diseases in men. Hum Mov 2010;11(1):45-50.

17. Cassani RSL, Nobre F, Pazin Filho A, Schmidt A. Prevalência de fatores de risco cardiovascular em trabalhadores de uma indústria brasileira. Arq Bras Cardiol 2009;92(1):16-22.

18. Nunes Filho JR, Debastiani D, Nunes AD, Peres KG. Prevalência de fatores de risco cardiovascular em adultos de Luzerna, Santa Catarina, 2006. Arq Bras Cardiol 2007;89(5):319-324.

19. Cavagioni LC, Benseñor IM, Halpern A, Pierin AMG. Síndrome metabólica em motoristas profissionais de transporte de cargas da rodovia BR-116 no trecho paulista - Régis Bittencourt. Arq Bras Endocrinol Metab 2008;52(6):1015-23.

20. Rezende FAC, Rosado LEFPL, Ribeiro RCL, Vidigal FC, Vasques ACJ, Bonard IS, et al. Índice de massa corporal e circunferência abdominal: associação com fatores de risco cardiovascular. Arq Bras Cardiol 2006;87(6):728-34.

21. Martinez MC, Latorre MRDO. Fatores de risco para hipertensão arterial e diabete melito em trabalhadores de empresa metalúrgica e siderúrgica. Arq Bras Cardiol 2006;87(4):471-9.

22. Polanczyk CA. Fatores de risco cardiovascular no Brasil: os próximos 50 anos! Arq Bras Cardiol 2005;84(3):199-201.

23. Vedana EHB, Peres MA, Neves J, Rocha GC, Longo GZ. Prevalência de obesidade e fatores potencialmente causais em adultos em região do sul do Brasil. Arq Bras Endocrinol Metab 2008;52(7):1156-62.

24. Paccini MK, Arsa G, Glaner MF. Indicadores de gordura abdominal: antropometria vs absortometria de raio-x de dupla energia. Rev Bras Cineantropom Desempenho Hum 2008;10(3):283-8.

25. Zhu S, Wang Z, Heshka S, Heo M, Faith MS, Heymsfield SB. Waist circumference and obesity-associated risk factors among whites in the Third National Health and Nutrition Examination Survey: clinical action thresholds. Am J Clin Nutr 2002;76(4):743-9.

26. Tarastchuk JCE, Guérios ÊE, Bueno RRL, Andrade PMP, Nercolini DC, Ferraz JGG, et al. Obesidade e intervenção coronariana: devemos continuar valorizando o índice de massa corpórea? Arq Bras Cardiol 2008;90(5):311-6.

27. Lima WA, Glaner MF. Body fat topography as a predictor of an increase in blood lipids. RBM, Rev bras med 2009; 1(suppl especial): 3-9. 
28. Wajchenberg BL. Subcutaneous and visceral adipose tissue: their relation to the metabolic syndrome. Endocrine Reviews 2000;21(6):697-738.

29. Deurenberg P, Yap M, van Staveren WA. Body mass index and percent body fat: a meta analysis among different ethnic groups. In J Obesity 1998;22(12):1164-71.
Endereço para correspondência

Maria Fátima Glaner

Quadra 201, Lote 6, Bloco B - apt. 803

CEP: 71937-540 - Águas Claras / Brasília - DF

E-mail: mfglaner@gmail.com 